Superalloys 2012: $12^{\text {th }}$ International Symposium on Superalloys Edited by: Eric S. Huron, Roger C. Reed, Mark C. Hardy, Michael J. Mills, Rick E. Montero, Pedro D. Portella, Jack Telesman TMS (The Minerals, Metals \& Materials Society), 2012

\title{
A NEW APPROACH TO MODELING OF CREEP IN SUPERALLOYS
}

\author{
Ramkumar Oruganti $^{1}$, Mallikarjun Karadge ${ }^{2}$, Sachin Nalawade ${ }^{1}$, Sivakumar Kelekanjeri ${ }^{1}$ and Francesco Mastromatteo ${ }^{3}$ \\ ${ }^{1} \mathrm{GE}$ Global Research, John F Welch Technology Center, Bangalore - 560066, India \\ ${ }^{2}$ GE Global Research, Niskayuna, NY-12309, USA \\ ${ }^{3}$ GE Oil and Gas, Nuovo Pignone, Florence, Italy
}

Keywords: Creep, superalloys, networks, climb, model, dislocations

\begin{abstract}
A new theory of superalloy creep is presented. It is argued that $\gamma^{\prime}$ particles in superalloys do not play a rate-controlling role during creep. They are hypothesized to act as composite reinforcements regardless of their size. The rate-controlling role is ascribed to dislocation related obstacles such as nodes, jogs or kinks. The basic constitutive law based on this premise is shown to be capable of representing the time to strain data of a wide range of superalloys. Equations for primary and tertiary creep are developed based on the composite concept and are shown to predict the creep behavior of DS-GTD $111^{\mathrm{TM}}$ a superalloy commonly used in gas turbines. The new approach is also shown to be capable of explaining a wide range of observations reported in the literature. The implications of these findings in terms of the effect of microstructure on creep behavior are discussed.
\end{abstract}

\section{Introduction}

The creep behavior of superalloys has been investigated extensively since their main use is in high temperature and high stress applications. The most common mechanism invoked in trying to explain superalloy creep is dislocation climb over particles. This is apparently motivated by two considerations, namely - 1) superalloys contain large amounts of second phase particles which perforce leads one to conclude that the particles must be impeding creep in some manner and 2) climb has been a commonly accepted mechanism for creep from the early work of Weertman [1] and more recently of Rosler and Arzt [2]. When microstructural analysis and creep data (strain rate vs. stress) do not support the climb concept, alternative mechanisms such as $\gamma^{\prime}$ shearing, Orowan looping, microtwining etc are offered as alternative mechanisms.

Climb is by far the most commonly invoked rate controlling mechanism in superalloy creep and the references for this are too numerous to list here. But no physical model based on this mechanism has been shown to describe the creep behavior of even a simple unimodal superalloy over a wide range of stress and temperature. A model by Dyson [3] that invokes climb, though not explicitly, in a rate controlling role has not been able to describe the creep behavior of Nimonic 90 at high temperatures and low stresses. (It is worth noting that many models for creep of superalloys report lack of match between experiment and predictions especially at high temperatures and low stresses [3, 4], though each study ascribes a different reason for the lack of concordance). One of the factors in this shortcoming appears to be the purported role of $\gamma$ ' spacing and size on creep. Climb based models generally assume that the interparticle spacing plays a key role in determining the creep response. Hence the creep resistance is expected to decrease with the increase in $\gamma^{\prime}$ spacing which occurs due to coarsening [3]. That this does not happen in reality can be illustrated with the aid of Fig. 1 which is a plot of stress vs. rupture time for 3 different superalloys on an LMP scale (Nimonic 90[3], IN738 [5], CMSX4 [4].

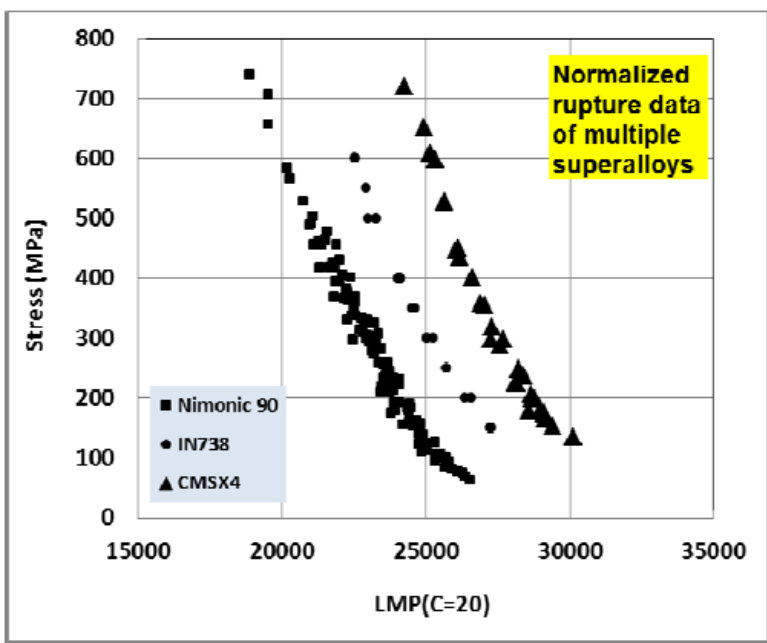

Fig. 1: Stress vs. LMP for different superalloys showing basic similarity in the shapes of the curves.

The Larson-Miller principle dictates that creep data, when plotted on a stress vs. LMP graph, should yield a straight line. Real materials generally deviate from linearity and important clues about the creep response can be gleaned from the nature of this deviation. For instance, if a material weakens with time due to coarsening of strengthening particles, the plot will reflect this by curving downward. A good example of this is the case of ferritic steels where nanoscale carbonitrides coarsen and weaken the material [6]. The data for superalloys, on the other hand, appear to curve upwards as shown in Fig. 1. The curvature also appears to be identical over many different superalloys and 
the data for any alloy can be obtained from that of any other alloy by simple scaling. Clearly an alternative explanation is required for the characteristic shapes of the curves shown in Fig. 1. One possibility is that the apparent increase in strength with time could be attributed to the increase in size of $\gamma$ ' which could make climb more difficult. But this explanation would require that different superalloys exhibit different slopes at higher values of LMP depending on the specific coarsening rates of $\gamma^{\prime}$. Obviously this is not the case and in fact the overall trends in slopes of the curves in Fig. 1 are almost identical. Hence the $\gamma$ ' particles appear to be playing some role that is not related to a rate-controlling process such as climb. The arguments presented here are not an attempt to negate the occurrence of climb during creep - clearly there is copious evidence of this phenomenon occurring during creep. It is only being stated that climb appears to be an unlikely candidate as a rate controlling mechanism even in simple unimodal superalloys.

A few early studies attempted a more critical examination of climb in greater detail as it applies to superalloys. Henderson and McLean [7] through friction stress measurements showed that $\gamma$ ' particle spacing and its increase due to coarsening did not seem to affect the creep behavior in IN738. They postulated that the dislocation networks surrounding the $\gamma$ 'particles probably controlled the creep behavior. Dyson and McLean [8], quoting Tipler and Peck [9] showed that the creep response of pre-aged and virgin IN738 did not show any difference when crept at a stress that resulted in a life of about 8000 hours. Thus, while superalloy technology has progressed considerably in the past decades, there clearly has been no consensus on the correct mechanism of creep in these important alloys.

More recently, TEM analyses of disk superalloys have shown drastically different dislocation structures involving the formation and propagation of stacking faults and microtwins [10]. There appear to be no hints of dislocation climb. Yet these materials appear follow the same stress vs. LMP trends shown show by all other superalloys. Thus it appears that a more fundamental mechanism could underlie creep and a mathematical description of this mechanism could explain the creep data of a wide range of superalloys.

In an effort to arrive at such a description, the current article takes an approach towards creep that is different from the climb-glide path taken hitherto and focuses more on the dislocations and their networks. It is postulated that $\gamma$ ' particles act fundamentally as composite reinforcements and have no rate-controlling role. A quantitative model based on this premise is developed below. It will also be shown that the model is able to consistently explain a wide range of data and also throw light on some observations reported in the literature.

\section{Model development}

Basic constitutive law

The fundamental starting point is the hypothesis that the rate controlling obstacles are not particle related but dislocation related. This perspective is dealt with fully in Ref. [6], but briefly, the main arguments are as follows:

a) As shown in Fig. 1 creep data for a range of superalloys follow similar trends. Such plots have been constructed for many other superalloys, though all of them are not shown here. While a fortuitous confluence of various mechanisms operating at various stress and temperature combinations could have occurred to cause this similarity, a much simpler explanation would be that a similar creep mechanism underlies all these materials.

b) TEM micrographs of crept modern superalloys show dense dislocation networks rather than individual dislocations climbing over particles. The latter picture is invoked in models of climb controlled creep [2] and clearly this is not observed in high volume fraction superalloys. This combined with the fact that the trends of creep data of modern and old superalloys are similar, points to the possibility that the rate controlling mechanism has not changed over many generations of superalloy development. This leads us to believe that the rate-controlling mechanism could be related to the dislocations and their networks rather than the particles.

c) Based on the above, $\gamma^{\prime}$ particles are assumed to act only as composite reinforcements with strengthening proportional to their volume fraction.

The assumption of a dislocation related rate controlling obstacle leads to a stress dependent activation volume which in turn leads to the following constitutive law relating creep rate to stress [6]:

$\dot{\varepsilon}=A \exp \left(-\frac{Q_{C}}{R T}\right) \sinh \left(S\left[\sigma-\sigma_{B}\right]^{n}\right)$

where $A$ and $S$ are constants, $Q_{C}$ is the activation energy for creep, $\sigma$ is the applied stress, $\sigma_{B}$ is the back-stress and $n$ is a constant. As a first approximation $\sigma_{B}$ is taken to be equal to $f \sigma$ where $f$ is the volume fraction of strengthening particles. In order to apply this equation to creep data generally found in the literature, we use the MonkmanGrant approach to invert the left hand side of Eq. 1 and write the time to attain a specified amount of strain as

$t=\frac{\exp \left(\frac{Q_{C}}{R T}\right)}{A \sinh \left[S\left(\sigma-\sigma_{B}\right)^{n}\right]}$ 
Fig. 2 shows fits to creep data of various superalloys plotted in the form of stress vs. time to rupture and Table 1 shows the values of the parameters. The value of the parameter $S$ is close to unity and is taken to be so for all the materials.

(a)

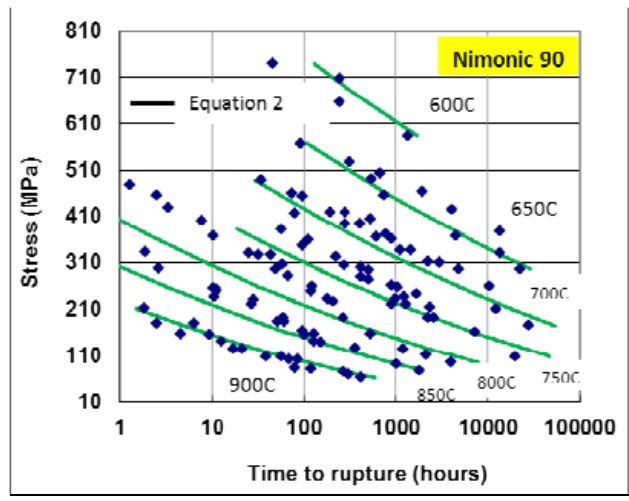

(b)

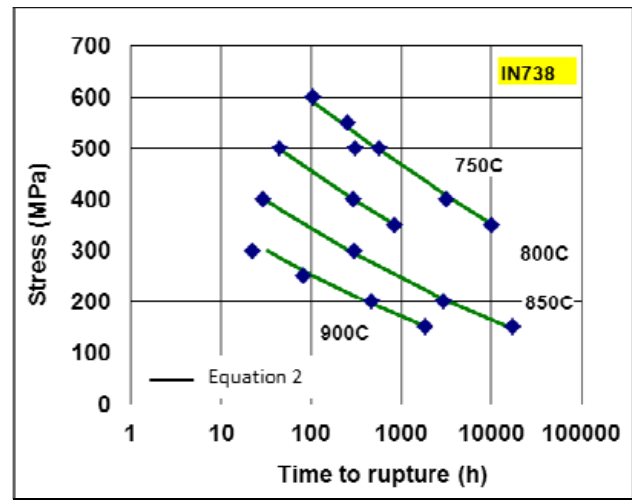

(c)

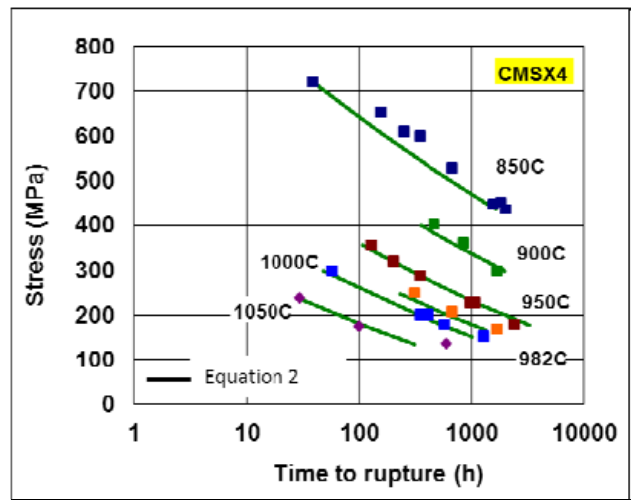

Fig. 2: Experimental and fit stress vs. time to rupture for three different superalloys, Nimonic90 [3], IN738 [5] and CMSX4 [4]. Eq. 2 is able to represent the creep behavior of all these superalloys very well.
The volume fraction of $\gamma^{\prime}$ was taken to be a function of temperature wherever such data was available. It is shown that Eq. 2 is able to represent the creep data of all these materials very well with the exponent $n$ being close to 0.5 for all of them. Fig. 2 includes data for superalloys that span various microstructural characteristics - grain morphology, $\gamma^{\prime}$ volume fraction and bimodal vs. unimodal $\gamma^{\prime}$. In spite of all these differences, the time to strain data are represented very well by a simple constitutive law and all of them have an exponent that is remarkably close to 0.5. This strongly supports the approach taken in this paper that the underlying mechanism that controls creep may be similar in all these materials. As shown in Ref. [6], n 0.5 appears to be applicable to many single phase materials also.

The main point to be noted here is that excellent description of the creep data was achieved without invoking complex creep and damage mechanisms. Usage of, what in our opinion is a simple yet appropriate constitutive law, leads to this interesting result.

Table 1: Parameters in Eq. 2 for three superalloys used for plotting graphs in Fig. 2.

\begin{tabular}{|l|l|l|l|l|}
\hline Alloy & $\boldsymbol{A}\left(\mathrm{h}^{-1}\right)$ & $\boldsymbol{Q}(\mathrm{kJ} / \mathrm{mol})$ & $\boldsymbol{n}$ & $\boldsymbol{f}\left(\boldsymbol{T}\right.$ in $\left.^{\circ} \mathbf{C}\right)$ \\
\hline Nimonic90 & $5.0 \mathrm{e} 12$ & 410 & 0.50 & $3.64 \mathrm{E}-11 \mathrm{~T}^{4}-$ \\
& & & & $1.09 \mathrm{E}-07 \mathrm{~T}^{3}+$ \\
& & & & $1.20 \mathrm{E}-04 \mathrm{~T}^{2}-$ \\
& & & & $0.0581 \mathrm{~T}+10.7$ \\
\hline IN738 & $2.5 \mathrm{e} 13$ & 460 & 0.52 & $\sim 0.5$ \\
\hline CMSX4 & $1.0 \mathrm{e} 13$ & 460 & 0.52 & $\sim 0.7$ \\
\hline
\end{tabular}

\section{Modeling the strain vs. time curve}

We now proceed to develop equations that enable modeling of the entire strain vs. time curve. As stated earlier, the main premise of this paper is that $\gamma$ ' particles do not play any rate-controlling role during creep. It is postulated that they primarily act as composite reinforcements. With this in mind, primary and tertiary creep are modeled as consequences of the interaction between the creeping matrix and the mostly non-deformable particles. The parameter that captures this interaction most effectively is the back stress ( $\sigma_{B}$ in Eq. 1).

Following Dyson [3] and Ion et al [11] we hypothesize that the process of generation of the back stress leads to primary creep. The back stress is generated when stress is transferred from the creeping matrix to the hard $\gamma^{\prime}$ particles. The following equation, proposed by Dyson [3] describes the evolution of back-stress

$\dot{\sigma}_{B}=f E \dot{\varepsilon}\left(1-\frac{\sigma_{B}}{H^{*} \sigma}\right)$ 
where $E$ is the elastic modulus of the $\gamma^{\prime}$ phase and $H^{*}$ is equal to $f /(1+2 f)$ where $f$ is the volume fraction of $\gamma^{\prime}$. We propose that it suffices to view $H^{*}$ as the simply equal to $f$ itself. The maximum value of the back stress is obtained by equating the right hand side of Eq. 3 to zero giving $\sigma_{B(\max )}$ $=f \sigma$. Thus the maximum back stress is purely a function of the volume fraction of the strengthening phase as stated previously.

Generation of back stress and the mechanism by which this happens has been dealt with in detail in literature related to composites [12,13]. Back stresses are generated when strain gradients between non-deformable particles and a deformable matrix have to be accommodated. This accommodation occurs by the generation of dislocations which lead to long range stresses in the matrix. Concomitantly, these stresses lead to elastic strains in the non-deformable particles. Thus, in a sense it may be stated that the dislocation structure acts a medium that transfers stress from the creeping matrix to the non-deforming particles. In the case of superalloys, it may be hypothesized that the dislocation network structure that forms around $\gamma$ ' particles as shown schematically in Fig. 3, transfers stress from the $\gamma$ matrix to the $\gamma$ ' particles. Such a view, apart from describing primary creep, also allows easy description of tertiary creep as explained below.

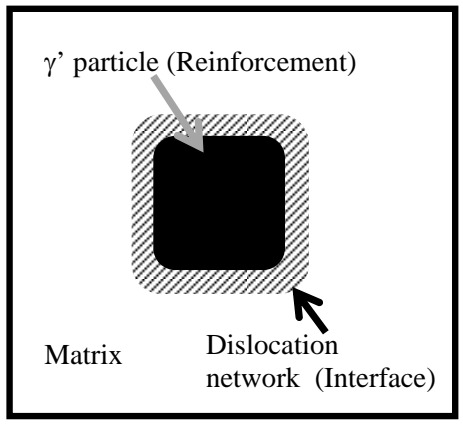

Fig. 3: Schematic showing single reinforcing $\gamma^{\prime}$ particle within a $\gamma$ matrix. The 'interface' allows stress transfer between the creeping matrix and the particle. The role of the interface is assigned to dislocation networks in the case of superalloys.

\section{Tertiary creep}

Based on the concept outlined above, it is postulated that tertiary creep occurs when the $\gamma$ ' particles or the interface (dislocation network) are no longer carrying/transferring stress as efficiently as they should. In other words, either the $\gamma^{\prime}$ or the interface is deforming. The former possibility (namely penetration of $\gamma$ ' by dislocations) has been noticed earlier as a phenomenon that appears to coincide with the onset of tertiary creep [14]. This is analogous to the case of composites where failure occurs when either the particles fracture or the notional particle-matrix interface is not able to accommodate the local strain gradients. The drop in back-stress due to these weakening mechanisms leads to an accelerating creep rate leading to failure. This view of tertiary creep is analogous to slipping interface models invoked to explain creep in composites [15].

The proposed concept for tertiary creep in superalloys is mathematically represented by defining an effective $\gamma$ ' load carrying fraction, $f_{e}$. This fraction decreases with increasing inability of the $\gamma^{\prime}$ particles or the interface to carry or transfer load, as the case may be. The rate of evolution of this current effective $\gamma^{\prime}$ volume fraction, $f_{e}$, is assumed to be a linear function of creep strain and is written as

$$
f_{e}=f\left[1-K_{f} \exp \left(-\frac{Q_{f}}{R T}\right) \varepsilon\right]
$$

where $\varepsilon$ is the macroscopic creep strain, $K_{f}$ is a proportionality constant, $f$ is the total $\gamma^{\prime}$ volume fraction and $Q_{f}$ is the activation energy corresponding to this damage mechanism. Thus the effective $\gamma$ ' volume fraction is assumed to decrease linearly with strain with a temperature dependence. A normalized damage parameter $D_{f}=f_{e} / f$ is defined such that it can be introduced into Eq. 3 as follows (assuming $H^{*}$ to be equal to $f$ )

$\dot{\sigma}_{B}=E \dot{\varepsilon}\left[f \cdot D_{f}-\frac{\sigma_{B}}{\sigma}\right]$

As tertiary creep proceeds, the effective load carrying fraction of $\gamma$ ' decreases continuously leading to a decreasing back-stress. This results in an accelerating creep rate.

According to the model presented here, the fundamental creep mechanism remains unchanged over all strains, stresses and temperatures. $\gamma$ ' shearing etc. are viewed purely as damage mechanisms which accelerate the basic creep process. Thus a clear demarcation is made between creep and damage, the lack of which we believe is the source of much confusion regarding the exact ratecontrolling mechanism in superalloy creep.

\section{Predictions and discussion}

Predictions of the creep behavior of DS-GTD $111^{\mathrm{TM}}$, a $\gamma^{\text {' }}$ strengthened superalloy with about 55 vol\% $\gamma^{\prime}$ are shown in Fig. 4. It is seen that all stages of creep are predicted fairly well by the set of equations resulting from the composite conjecture. Thus a simple physical picture of stress transfer to $\gamma$ ' particles causing primary creep and subsequent shedding of this stress leading to tertiary creep is able to explain most features of creep in superalloys. In this picture, the rate controlling role is played by dislocation 
related obstacles and the characteristics of these obstacles could depend on factors like misfit, diffusivity and segregation of key elements. These factors are currently implicitly included in the various parameters in Eqs. 1, 4 and 5 and further work is required to tease out these underlying relationships.

\section{Corollaries}

1) The composite theory of $\gamma^{\prime}$ strengthening instructs us that secondary $\gamma^{\prime}$ size and spacing should have no effect on creep resistance, similar to the case of composites. The following experiment was carried out to verify this effect. Samples of DS-GTD $111^{\mathrm{TM}}$ with different initial secondary $\gamma$ ' sizes were tested under various creep conditions. One set of samples had an initial average $\gamma$ ' size of $607 \mathrm{~nm}$ while the other had a size of $815 \mathrm{~nm}$. It was ensured that the sizes were sufficiently far apart that one would not catch up with the other during the creep tests. The data from these tests is shown in Fig. 5. It is clear from the plot that coarsening of the secondary $\gamma$ ' had no effect on the creep behavior under the range of stress considered here.

If $\gamma^{\prime}$ spacing is extremely small, as in the case of high volume fraction superalloys, additional composite strengthening could arise from constraint effects. Though this will have a dependence on spacing, it does not detract from the basic principle that the particles are not ratecontrolling in any sense.

2) Following from the earlier point on high volume fraction superalloys, it may be stated that the major influence of rafting would be twofold. The first would be when the fine interparticle spacings are lost in the initial stages leading to loss of additional constraint effects arising from closely spaced particles. The second would be when all the vertical channels are completely closed and the composite becomes infinitely hard due to lack of avenues for dislocations to move and cause deformation. In the latter case, creep strain vs. time curves show flat behavior with very little strain accumulation. Between these two extremes, incomplete rafts still behave as composite reinforcements and probably have no other significant role to play with respect to creep deformation. We believe that this is the reason for the excellent fits obtained for the creep data of CMSX4 using Eq. 2 as shown in Fig. 2c. CMSX4 is known to raft even at $950^{\circ} \mathrm{C}$ and this does not affect the ability of Eq. 2 to represent the high temperature creep data. The only data point that lies outside the fits is at the longest time at $1050^{\circ} \mathrm{C}$ and it is likely that this data point corresponds to completion of rafting. This is a point that requires further study.

Interpretation of neutron scattering studies

The composite concept proposed here to explain superalloy creep is also able to throw light on the results of the neutron
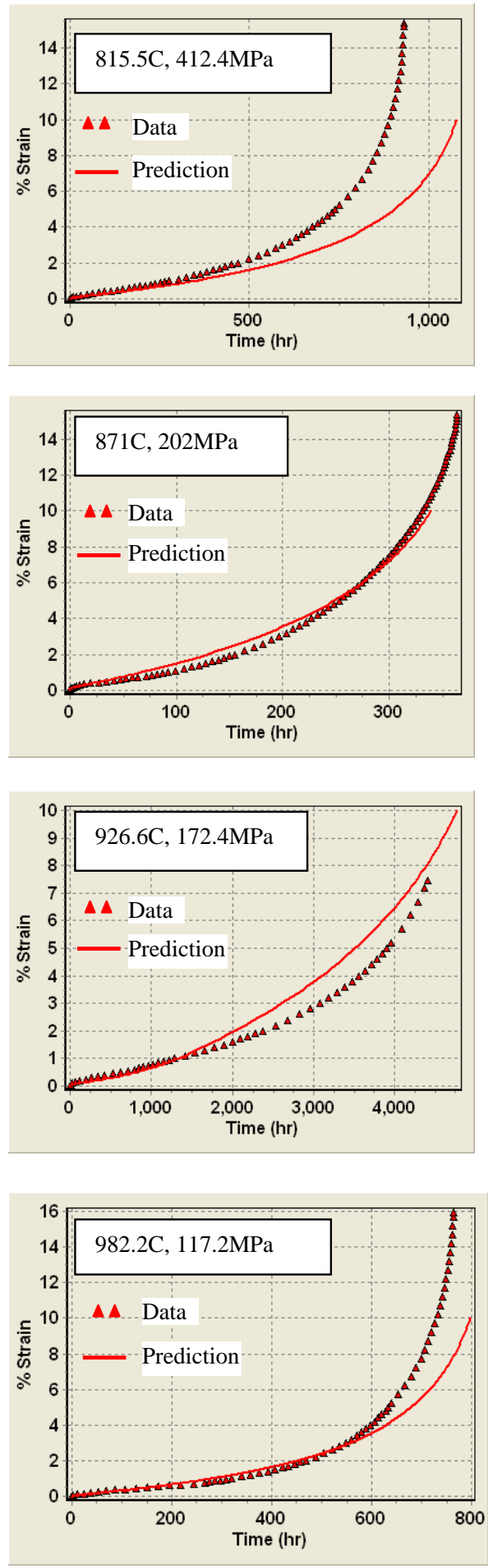

Fig. 4 Experimental and predicted strain vs. time curves for DS-GTD $111^{\mathrm{TM}}$ at various time-temperature combinations 
diffraction studies of Ma et al [16] and Preuss et al [17]. Ma et al carried out constant strain rate experiments (which can be used to interpret creep mechanisms) while observing the elastic strains within the $\gamma$ and $\gamma$ ' phases. They observed that the elastic strain within the $\gamma^{\prime}$ phase increased up to a point beyond which it decreased. In light of the earlier discussions, the increase in $\gamma^{\prime}$ elastic strain may be interpreted as reflecting a process of stress transfer to the $\gamma$ ' particles. Upon reaching a maximum the particles begin to shed stress leading to tertiary creep. This shedding may be a consequence of particle cutting or breakdown of the interface. Both of these mechanisms lead to decrease in the effective stress carrying volume fraction of $\gamma$ '. Preuss et al in their experiments observed that stress partitioning between $\gamma$ and $\gamma$ ' appeared to depend on the fineness and distribution of the $\gamma$ 'particles. A material with a finer distribution appeared to show less stress partitioning than a material with a coarser distribution, especially at elevated temperatures. They correlated this to the observation of shearing of the $\gamma^{\prime}$ and concluded that shearing precluded the transfer of stress to the particles. Another way of viewing this result is to observe that the finer microstructure would suppress percolation of dislocations within the channels. Thus a few particles would end up carrying a significant portion of the applied stress and subsequently undergo shearing due to this stress build- up. This would probably not be detectable by neutron scattering.

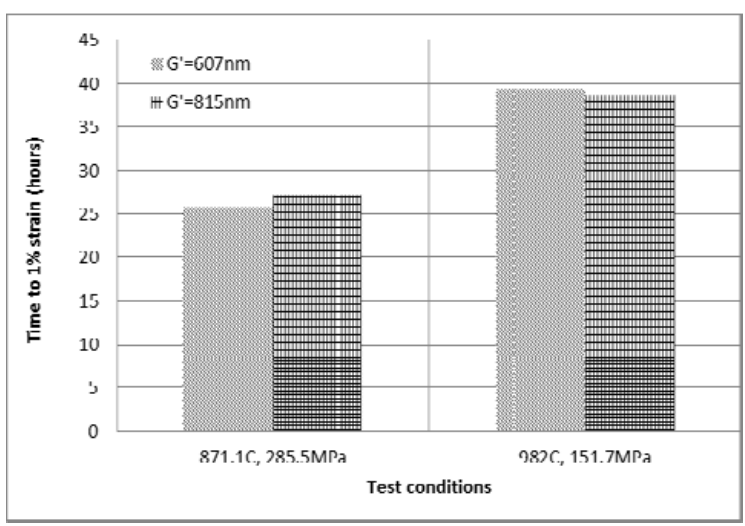

Fig. 5: Time to $1 \%$ strain for different initial secondary $\gamma$ ' sizes. Data shows no dependence of the former on the latter.

\section{Summary and conclusions}

1. A new constitutive equation for superalloy creep has been proposed. The basic premise underlying this equation is that $\gamma^{\prime}$ particles are not rate controlling obstacles for creep in superalloys. This role is assigned to dislocation related obstacles such as nodes, jogs or kinks. $\gamma$ ' particles, regardless of their size are hypothesized to act as composite reinforcements.

2. A new interpretation of dislocations as 'interfaces that transfer load' is proposed. This allows calculation of potential strengthening from $\gamma^{\prime}$ particles.

3. Primary and tertiary creep are modeled solely based on the load transfer mechanism and its decreasing efficacy with increasing strains.

4. The fundamental constitutive equation is shown to be capable of representing the creep response of a range of superalloys. The full equation set comprising only three simple equations is shown to be capable of explaining the creep behavior of DS-GTD $111^{\mathrm{TM}}$ over a wide range of temperatures and stresses.

5. It is proposed that creep and damage mechanisms should not be conflated. The former remains unchanged while the latter can change with strain, stress and temperature (in intra-grain creep).

6. The main implication of the approach presented here is that the phenomenon of creep in superalloys can be distilled down to a few essential elements. The fundamental reason for increases in creep resistance of successive generations of superalloys can probably be traced to slowing down of what is essentially a common rate-controlling process.

\section{Acknowledgements}

Funding for this research was provided by GE Global Research's Advanced Technology Program on Microstructure Based Lifing, managed by Dr. Margaret Blohm. Additional funding and some experimental data were provided by GE Oil and Gas. Discussions with Dr. Sanjay Sondhi, Dr. Mahesh Chandran, Dr. Srinivasan Swaminathan and T. Vishwanath, Scientists at GE Global Research are gratefully acknowledged. Thanks are due to Dr. Swapnil Patil of GE Global Research for a thorough perusal of the manuscript which led to some improvements. Thanks are also due to Dr. Brian Dyson for stimulating discussions and useful inputs on the fundamental nature of creep

\section{References}

[1] J. Weertman, "Theory of steady state creep based on dislocation climb”, Journal of Applied Physics, Vol. 26, 1955, 1213.

[2] J. Rosler J and E. Arzt, "The kinetics of dislocation climb over hard particles - I. Climb without attractive particle-dislocation interaction”, Acta Metall 1998, Vol. 36, 1043-1051

[3] Dyson BF. "Microstructure based creep constitutive model for precipitation strengthened alloys - theory and 
application”, Materials Science and Technology, Vol. 25, 2009, 213-220.

[4] A. Ma, D. Dye and R.C. Reed, "A model for the creep deformation behavior of single crystal superalloy CMSX4”, Acta Materialia, Vol. 56, 2008, 1657-1670.

[5] NIMS datasheets, http://mits.nims.go.jp/en/

[6] R. Oruganti, “A New Approach to Dislocation Creep”, Acta Materialia, Vol. 60, 2012, 1695-1702.

[7] P. J. Henderson and M.McLean, "Microstructural Contributions to Friction Stress and Recovery Kinetics During Creep of the Nickel-Base Superalloy IN738LC", Acta Metallurgica, Vol. 31, No. 8, 1983, 1203-1219.

[8] B.F.Dyson and M. McLean, "Particle Coarsening, $\sigma_{0}$ and Tertiary Creep”, Acta Metallurgica, Vol. 31, 1983, $17-$ 27.

[9] H.R. Tipler and M.S. Peck, Final Report on COST 50 Programme UK 17, Division of Materials Applications, National Physical Laboratory, Teddington, U.K. DMA(A)33(1981).

[10] G.B. Viswanathan et al, "Investigation of creep deformation mechanisms at intermediate temperatures in René 88 DT”, Acta Materialia, Vol. 53, 2005, pp. 30413057.

[11] Ion, J. C., Barbosa, A., Ashby, M. F., Dyson, B. F., and McLean, M., 1986, "'The Modelling of Creep for Engineering Design-I,’” NPL Report DMAA115, The National Physical Laboratory, Teddington, Middesex, U.K.

[12] S. Goto and M. McLean, " Role of interfaces in creep of Fibre-reinforced metal-matrix composites-I. Continuous fibres', Acta Metallurgica et Materialia, Vol. 39, 1991, pp. 153-164.

[13] H. Lilholt, "Strengthening and internal stresses in composites under mechanical and thermal loading”, in Mechanical and Physical Behavior of Metallic and Ceramic Composites, Eds. S.I. Anderson, H. Lilholt and O.B. Pederson, 1988, Riso National Laboratory, Rosskilde, Denmark, pp. 89-107.

[14] T.M.Pollock and A.S. Argon, "Creep resistance of CMSX-3 Nickel base superalloy single crystals”, Acta Metallurgica et Materialia, Vol. 40, 1992, pp. 1-30.

[15] Prasad B. Nimmagadda and P. Sofronis, "Creep strength of fiber and particulate composite materials: The effect of interface slip and diffusion”, Mechanics of Materials, Vol. 23, 1996, 1-19.
[16] S. Ma et al 'CRSS of $\gamma / \gamma$ ' phases from in situ neutron diffraction of a directionally solidified superalloy tension tested at $900^{\circ} \mathrm{C}^{\prime}$, Acta Materialia, Vol. 56, 2008, 41024113.

[17] Michael Preuss et al, “The Effect of $\gamma$ ' Particle Size on the Deformation Mechanism in an Advanced Polycrystalline Nickel-Base Superalloy”, Superalloys 2008, eds. Roger C. Reed et al (TMS, Warrendale, Pa, USA), 405-414. 